\title{
Quality Matters: A Global Discussion in Qatar
}

\author{
Ayat Salman, ${ }^{1-3,{ }^{*}}$ Ronny Baber, ${ }^{3-5,{ }^{*}}$ Linda Hannigan, ${ }^{6}$ Jens Karsten Habermann, $, 3,8$ \\ Marianne K. Henderson, ${ }^{9,10}$ Michaela Th. Mayrhofer, ${ }^{11}$ and Nahla Afifi ${ }^{6}$
}

The International Biobanking Conference titled “Quality Matters: A Global Discussion in Qatar" was held on March 25-27, 2019, in the vibrant city of Doha, Qatar. The 3-day event was organized and hosted by the Qatar Biobank (QBB) and the European, Middle Eastern and African Society for Biopreservation and Biobanking (ESBB), with supporting collaboration from the International Society for Biological and Environmental Repositories (ISBER) and the Biobanking and BioMolecular Resources Research Infrastructure-European Research Infrastructure Consortium (BBMRI-ERIC). The aim was to highlight the role of biobanking in medical research and advancing health care, as well as improving clinical outcomes. The conference convened experts from across the globe to discuss continuing efforts to harmonize biobanking-related processes to achieve high-quality standards and to support international advancements in medical research for our diverse populations. The scientific agenda drew more than 1000 scientists, researchers, industry experts, and health professionals from five continents. The conference focused on the quality aspect of biobanking through seven sessions over 3 days. Researchers, scientists, and experts from around the world were invited to present, and included special presentations from QBB demonstrating their standing as a leading clinical biobank innovator in support of population and genomic medicine. The 3-day conference concluded with a session on Best Practices and Standards, a topic much in discussion with today's context.

Keywords: biobank, conference, editorial, Qatar biobank

\section{Quality Matters: A Global Discussion in Qatar}

$\mathrm{T}$ He International Biobanking Conference titled "Quality Matters: A Global Discussion in Qatar" was held on March 25-27, 2019, in the vibrant city of Doha, Qatar. The 3-day event was organized and hosted by the Qatar Biobank (QBB) and the European, Middle Eastern and African Society for Biopreservation and Biobanking (ESBB), with supporting collaboration from the International Society for Biological and Environmental Repositories
(ISBER) and the Biobanking and BioMolecular Resources Research Infrastructure-European Research Infrastructure Consortium (BBMRI-ERIC). The aim was to highlight the role of biobanking in medical research and advancing health care, as well as improving clinical outcomes. The value that biobanks offer globally to the advancement of precision medicine should not be undervalued. The conference convened experts from across the globe to discuss continuing efforts to harmonize biobanking-related processes to achieve high-quality standards and to support

${ }^{1}$ McGill University Health Center, Royal Victoria Hospital (Glen), Quebec, Canada.

${ }^{2}$ Department of Family Medicine, McGill University, Quebec, Canada.

${ }^{3}$ ESBB Council, European, Middle Eastern and African Society for Biopreservation and Biobanking (ESBB), Brussels, Belgium.

${ }^{4}$ Institute of Laboratory Medicine, Clinical Chemistry and Molecular Diagnostics, University of Leipzig Medical Center, Leipzig, Germany.

${ }^{5}$ Leipzig Medical Biobank, University Leipzig, Leipzig, Germany.

${ }^{6}$ Qatar Biobank, Qatar Foundation, Doha, Qatar.

${ }^{7}$ Interdisciplinary Center for Biobanking-Lübeck (ICB-L), University of Lübeck, Lübeck, Germany.

${ }^{8}$ Section for Translational Surgical Oncology and Biobanking, Department of Surgery, University of Lübeck and University Hospital Schleswig-Holstein, Campus Lübeck, Lübeck, Germany.

${ }^{9}$ National Cancer Institute, National Institutes of Health, DHHS, Bethesda, MD.

${ }^{10}$ ESBB Council, International Society for Biological and Environmental Repositories (ISBER), Vancouver, Canada.

${ }^{11}$ ELSI Services Department, Biobanking and BioMolecular Resources Research Infrastructure-European Research Infrastructure Consortium (BBMRI-ERIC) Graz, Austria.

*Sharing first authorship. 
international advancements in medical research for our diverse populations. Key features of the meeting were presentations from QBB demonstrating their standing as a leading clinical biobank innovator in support of population and genomic medicine. QBB was established with a solid business model to meet the needs of the local research community.

The scientific agenda drew more than 1000 scientists, researchers, industry experts, and health professionals from five continents.

The conference focused on the quality aspect of biobanking through seven sessions over 3 days:

1. International Collaboration for Quality Results-Samples from Biobanks as Pacemaker for a Better Medicine;

2. Population-Based Biobanks: Learning from the Past and Targeting the Future;

3. Donor Matters: Incidental Findings and the Return of Genetic Data (supported by ISBER);

4. Pre-Analytics as a Key for Reproducibility;

5. Biobanks in Healthcare-from Basic Research to Clinical Use;

6. A Reach for Quality: Best practices and Standards (supported by ISBER);

7. Opportunities and Challenges of Big Data and Artificial Intelligence in Medical Research (supported by BBMRIERIC).

More than 65 abstracts were submitted to the scientific committee and presented as posters and flash talks. The poster session was open throughout the conference days.

Opening remarks were given by Dr. Richard O'Kennedy, Vice President of Research, Development, and Innovation at Qatar Foundation (QF-RDI), highlighting Qatar Foundation's research strategy, and its array of initiatives, designed to enable innovative health and medical research in Qatar. Dr. O'Kennedy underlined the transformation of biomedical research in precision medicine, "Biomedical research is accelerating the transformation of healthcare from generalized treatments to preventive, pre-emptive, and individualized medicine." Dr. O'Kennedy has played a crucial leading role within the important initiatives led by QBB and the Qatar Genome Program. The collaborating society opening statements were made by Prof. Jens K. Habermann, President of ESBB; Dr. Zisis Kozlakidis, Immediate Past President of ISBER; and Dr. Michaela Th. Mayrhofer, Head of Ethical Legal and Social Implication (ELSI) Services and Research of BBMRI-ERIC. Among the distinguished guests, the conference was honored to welcome the attendance of Dr. Hanan Al Kuwari, Minister of Public Health, Qatar, to the opening ceremony.

The scientific program began with keynote speaker, Dr. Jim Vaught, Editor-in-Chief of Biopreservation and Biobanking, the official journal of ISBER. He discussed "The Importance of Quality Management for International Collaborations," covering key topics in quality management, audits, preanalytical variations, and the complexities of quality control associated with biospecimens. In keeping with the main focus of the conference, Dr. Vaught completed his keynote presentation by reminding the audience of the importance of documenting biobank processes (e.g., Biospecimen Reporting for Improved Study Quality [BRISQ] and Standard PREanalytical Code [SPREC]) in publications to harmonize quality management across sites as well as the implementation of certification and accreditation of laboratories and biobanks.

The first session, entitled International Collaboration for Quality Results-Samples from Biobanks as a Pacemaker for a Better Medicine, focused on the importance of international collaborations in accelerating the advancement of precision medicine. The importance of harmonization in science, policy, and data governance was discussed as well as the importance of ethics and opportunities from sharing research outcomes. The session was chaired by Ms. Ayat Salman $^{1}$ and Dr. Reem Jawad Al Sulaiman. ${ }^{2}$ The session opened with local and international speakers sharing their experiences of national and international collaborations. The session included a panel discussion that highlighted some of the current genetic screening programs in Qatar collaborating with $\mathrm{QBB}$, and how these programs are pivotal in the development of modern technology around health services and policies for Qatar in the era of precision medicine.

The session opened with Prof. Jens K. Habermann ${ }^{3}$ presenting how clinical biobanks and strategic partnerships can support precision medicine, from the ESBB and Lübeck perspective. This was followed by presentations from Prof. Olli Carpen, ${ }^{4}$ Dr. Vasiliki Rahimzadeh, ${ }^{5}$ Dr. Mohammed Jarrar, ${ }^{6}$ Dr. Tawfeg Ben Omran, ${ }^{7}$ Dr. Salha Bujassoum Al Bader, ${ }^{8}$ and Dr. Samya Ahmad Al Abdulla. ${ }^{9}$ Their presentations covered a number of topics starting with the Finnish perspective of how population and hospital-based biobanks are enabling precision medicine, followed by Dr. Rahimzadeh's presentation stressing the importance of scientific collaboration and policy harmonization in data governance. Local presenters discussed various topics such as the implementation of genetic screening programs collaborating with QBB and the impact of this work on genetic screening programs; the improvement of cancer care in Qatar through precision medicine; and an insight into the work of the Primary Health Care Corporation (PHCC) in implementing screening programs for the Qatari population. Leading into the panel discussion, the final presentation was given by Dr. Roberto Bertollini, ${ }^{10}$ with a focus on the use of biobank data in public health research.

The second session, entitled Population-Based Biobanks: Learning from the Past and Targeting the Future, was chaired by Prof. Jens K. Habermann and Dr. Richard O'Kennedy. The session focused on the role of population-based biobanks and

${ }^{1}$ Doctorate Candidate at McGill University in the Department of Family Medicine and Manager MUHC HPB Clinical Research Group, Quebec, Canada.

${ }^{2}$ Professor of Genetics at Hamad Medical Corporation, Qatar.

${ }^{3}$ Director of the Interdisciplinary Center for Biobanking-Lübeck (ICB-L) at the University of Lübeck and University Clinic Schleswig-Holstein, Germany.

${ }^{4}$ Professor of Pathology at the University of Helsinki and Scientific Director at Helsinki Biobank, Finland.

${ }^{5}$ Post Doc Fellow at Stanford Center for Biomedical Ethics, Stanford University School of Medicine, Stanford, CA.

${ }^{6}$ Senior Manager of Biomedical and Health Research at Qatar National Research Fund, Qatar.

${ }^{7}$ Head of Clinical and Metabolic Genomics, Hamad Medical Corporation, Qatar.

${ }^{8}$ Senior Consultant at the National Center for Cancer Care and Research (NCCCR), Qatar.

${ }^{9}$ Executive Director of Operations and Senior Consultant Family Physician, Primary Health Care Corporation, Qatar.

${ }^{10}$ Advisor to the Minister of Public Health of Qatar, Qatar. 
their contributions in understanding the different genetic, environmental, occupational, and lifestyle risk factors of disease in populations. The session speakers highlighted accomplishments from the past and present, as well as how to integrate knowledge learned into prevention and precision medicine strategies for future populations. Dr. Asmaa Al Thani ${ }^{11}$ opened this afternoon session and presented the work of the QBB and the Qatar Genome Program and how they are taking major steps in implementing precision medicine in Qatar. Prof. Gillian Bartlett ${ }^{12}$ discussed the emerging epidemic of cardiometabolic disease and the importance of primary carebased biobanks contributions. Ms. Marianne K. Henderson ${ }^{13}$ spoke about population cohorts in the United States, the findings that have advanced our understanding of diseases, current challenges, and provided examples of next generation cohorts being launched for future research. The last presentation in this session was from Dr. Ronny Baber ${ }^{14}$ who shared experiences from the LIFE Project and how results from population-based biobanking can be used for clinical care.

The first day of the conference was concluded with a comprehensive guided tour of the QBB facilities. QBB highlighted the advanced monitoring tools that they have in place to support their population biobank participants.

Day 2 of the scientific program commenced with Dr. Zhengming Chen, Principal Investigator of the China Kadoorie Biobank, presenting his keynote lecture, "Realizing the Power of Large Biobanks in Diverse Populations," a fascinating overview of the research conducted by the China Kadoorie Biobank, one of the world's largest biobanks, with 500,000 Chinese participants. Dr. Chen presented the research into chronic diseases, such as diabetes, heart disease, and metabolic syndrome, some of the leading causes of death worldwide, supported by biospecimens collected in the China Kadoorie Biobank. Understanding the different causes of these diseases in diverse populations with different lifestyles, environments, and genetic backgrounds can lead to improved risk prediction, disease prevention, and the development of strategies for precision medicine in a primary care setting. Unique opportunities to fulfill these goals are offered by prospective "biobank" studies, with detailed characterization of large numbers of individuals from the general population, using conventional and novel technologies, and electronic monitoring of their health status.

The third scientific session, chaired by Dr. Zisis Kozlakidis ${ }^{15}$ and Dr. Eman Sadoun, ${ }^{16}$ focused on the hot topic of returning research results to biobank participants. The session, entitled Donor Matters: Incidental Findings and the Return of Genetic Data, highlighted the return of research data to study participants in many global contexts. The session featured local and international speakers sharing their

\footnotetext{
${ }^{11}$ Professor of Virology at the Biomedical Science Department, College of Health Sciences, Qatar University, Qatar.

${ }^{12}$ Tenured Professor, Research and Graduate Programs Director, and Associate Chair in the Department of Family Medicine at McGill University, Montreal, Canada.

${ }^{13}$ Senior Advisor for Division Resources, Division of Cancer Epidemiology and Genetics and Senior Advisor on Biobanking, Center for Global Health, Washington, DC.

${ }^{14}$ Head of LIFE-Biobank and Preanalytical Laboratory, University of Leipzig, Germany.

${ }^{15}$ Head of Laboratory and Biobanking Services IARC/WHO, France. ${ }^{16}$ Manager of Human Research at the Ministry of Public Health, Qatar.
}

experiences on the topic and concluded with a panel discussion with audience questions. Ms. Cristina Villena Portella ${ }^{17}$ opened the session and shared experiences of the Spanish Biobank Network with incidental findings and return of genetic data. Experiences in identifying, managing, and sharing incidental findings with its participants in Qatar, as well as shared experiences of the process of communicating with donors in the Graz Biobank were presented, respectively, by Prof. Nahla Afifi ${ }^{18}$ and Dr. Karine Sargsyan. ${ }^{19}$ Ms. Helena Judge Ellis ${ }^{20}$ followed with a presentation on what returning research results could mean for individual study participants considering the legal, ethical, and social issues. Dr. Khalid Al Ansari $^{21}$ closed out this session with his discussion about the local experience at Sidra Medicine in Qatar of identifying and returning incidental findings to patients.

Chaired by Ing. Pasquale De Blasio ${ }^{22}$ and Dr. Mohammed Ben Gashir, ${ }^{23}$ the session, entitled Pre-Analytics as a Key for Reproducibility, focused on the major impact that preanalytical variability can have on results of analyses of biospecimens. This was followed by a discussion panel on quality processes in a biobank and the collection of biological samples. Prof. Kurt Zatloukal ${ }^{24}$ opened the session presenting preanalytical issues in biobanking affecting medical research and diagnostics. Dr. Andrew Brooks ${ }^{25}$ spoke about the integration of new technology processes and quality control to protect the complete sample chain of custody. Dr. Ronny Baber spoke about pre-analytical conditions during long-term storage and their effects on the biostability of clinically relevant biomarkers. Dr. Jalaluddin Bhuiyan ${ }^{26}$ discussed the key to quality within the clinical laboratories and his journey to improved patient care in Hamad Medical Corporation. Kiran Govanidan-Kutty ${ }^{27}$ closed the session with his presentation on results of a systematic study into pre-analytical phlebotomy processes, its evaluation followed by active and passive interventions at the PHCC.

The concluding day started with a session entitled Biobanks in Healthcare, from Basic Research to Clinical Use. This session focused on the role of biobanks in the development of translational sciences and the impact of recent technological developments on precision medicine and was chaired by Prof. Kurt Zatloukal and Dr. Omar Al Bagha. ${ }^{28}$ Dr. Stephen Hewitt ${ }^{29}$ opened the session with his presentation

\footnotetext{
${ }^{17}$ Biobank Manager of Pulmonary Biobank Consortium CIBERES (CIBER-ISCIII), Spain.

${ }^{18}$ Director of Qatar Biobank, Qatar.

${ }^{19}$ Managing Director of Biobank Graz, Austria.

${ }^{20}$ Founder and Managing Director of Biobanking without Borders, Durham, NC.

${ }^{21}$ MD, FRCP-C, FAAP (PEM), Chair, Emergency Department, Sidra Medicine Professor, Weill Cornell Medical College, Qatar.

${ }^{22}$ Managing Director of ISENET, Milan, Italy.

${ }^{23}$ Consultant Pathologist with Hamad Medical Corporation, Qatar.

${ }^{24}$ Professor of Pathology at the Medical University of Graz and Head of the Diagnostic and Research Center for Molecular BioMedicine, Austria.

${ }^{25}$ Chief Operating Officer and Director of Technology Development of RUCDR Infinite Biologics at Rutgers University, NJ.

${ }^{26}$ DABCC, FAACC, Senior Consultant Clinical Scientist and Head of Laboratory Services, Hamad Medical Corporation, Qatar.

${ }^{27}$ Laboratory Quality Manager in Primary Health Care Corporation, Qatar.

${ }^{28}$ Principal Investigator with Qatar Biomedical Research Institute, Qatar.

${ }^{29}$ Clinical Investigator within the Laboratory of Pathology, National Cancer Institute, USA.
} 
on leveraging clinical samples in translational research, where he discussed the emphasis of reproducibility in science. $\mathrm{He}$ was followed by Prof. Christoph Brochhausen ${ }^{30}$ with his proposition of a road map to data-rich biobank specimens via digitalization in an international network. To wrap up the idea around the use of new technologies in biobanks, Ing. Pasquale De Blasio shared his vision of the next generation biobanking in keeping with the idea of reproducibility in science and the advancement of precision medicine. Following the same format as the previous sessions, experts from Qatar presented their work around the idea of the use of new technologies in precision medicine. Dr. Said Ismail ${ }^{31}$ and Dr. Ammira Akil, ${ }^{32}$ as well as Prof. Stephen Atkin, ${ }^{33}$ presented ongoing projects around the use of new technologies and data of QBB on translating research discoveries into the clinic.

Session 6 focused on best practices and standards in biobanking. The session was entitled A Reach for Quality: Best Practices and Standards and chaired by Prof. Nahla Afifi and Ms. Marianne Henderson. With the introduction of the ISBER fourth edition of Best Practices for Repositories, the newly released ISO Biobanking standard ISO 20837 and the emerging field of biorepository and biospecimen science, biorepositories worldwide are moving toward standardization. Dr. Daniel Simeon-Dubach ${ }^{34}$ opened the session with his presentation highlighting the tools available to the biobanking community in ISBERs latest edition of Best Practices to facilitate Quality Management of Biobanks. This was followed by Ms. Clare Alloca, ${ }^{35}$ who discussed A Path to Quality That Is Fit for Purpose, which included the scope and potential implementation of the ISO 20837 standard. This was followed by Dr. Zisis Kozlakidis, who presented A Reach for Quality: Best Practices and Standards in supporting the precision medicine vision. Dr. Michaela Th. Mayrhofer presented, on behalf of the quality management team of BBMRI-ERIC, the opportunities and current challenges of agreeing on harmonizing and implementing European and international standards, guidelines, and best practices relevant for biobanking and biomedical research. BBMRI-ERICs widely recognized Quality Management Services aims to support European biobankers and researchers in their mission to provide research excellence based on quality-assured samples and data. Qatar's view on standardization and the use of best practices in biobanking closed this session with a presentation by Ms. Fatima Qafoud. ${ }^{36}$ She presented the technical and operational best practices implemented and challenges faced at QBB.

The seventh and final session focusing on the future of medicine, entitled Opportunities and Challenges of Big Data and Artificial Intelligence in Medical Research, was chaired by Dr. Michaela Th. Mayrhofer and Dr. Said Ismail. Dr. Ahmed Elmagarmid $^{37}$ highlighted the vision and missions of the Na-

\footnotetext{
${ }^{30}$ Vice Chair of Pathology, Department of Pathology at the University Regensburg, Germany.

${ }^{31}$ Qatar Genome Project, Programme Manager, Qatar.

${ }^{32}$ Principal Investigator at Precision Medicine Program, Diabetes and Metabolism Program, Sidra Medicine, Qatar.

${ }^{33}$ Professor of Medicine at Weil Cornell, Qatar.

${ }^{34} \mathrm{MD}$, MHA, Biobank Consultant, Chair, ISBER Standards Committee, Switzerland.

${ }^{35}$ Senior Advisor for Standardization National Institute of Standards and Technology, Gaithersburg, MD.

${ }^{36}$ Clinic and Laboratory Manager, Qatar Biobank, Qatar.

${ }^{37}$ Founding Executive Director of the Qatar Computing Research Institute, Qatar.
}

tional Artificial Intelligence Strategy for Qatar. He was followed by Dr. Gianluigi Zanetti, ${ }^{38}$ who discussed the capture of simplified sample tracking information from acquisition to analysis, efficiently and reliably to make it meaningful to clinical researchers. He also described solutions to technical hurdles to drastically simplify and empower clinical researchers to harness modern cloud-based infrastructure for complete reproducibility and shareability of data intensive analyses. Mr. David Brown ${ }^{39}$ presented the challenges around the analysis of health system wide clinical and operational data with specific focus on the impact of linking genomic and clinical data. Dr. Radja Messai-Badji ${ }^{40}$ presented the importance of effective data governance and management in the conduct of biomedical research. The final presentation from Dr. Puthen Jithesh ${ }^{41}$ discussed how bioinformatics is playing a major role in translating the information hidden in big data sets to facilitate the implementation of pharmacogenomics for precision medicine.

Prof. Nahla Afifi and Prof. Jens K. Habermann concluded the 3-day conference with their closing remarks. Prof. Habermann commented on the conference and the progress that Qatar has made in biobanking and genomics and said, "the global discussion in Qatar is timely and important irrespective of which area of biobanking we each work in. We are here to ensure we excel in our capacities in biobanking in support of precision medicine globally."

Prof. Nahla Afifi believes that meetings, such as this International Biobanking Conference, can have a lasting impact: “This conference was an opportunity for Qatar's researchers and scientists to share their research and progress with international experts and share the best practices in the field; a crucial step to the success of large-scale biobanking endeavors in the region and internationally. We take firm steps every day, as we get closer to achieving precision medicine for the people of Qatar, which will prove vital in the treatment and prevention of diseases. The support we are receiving for our research programs, from our stakeholders, scientists, and the Qatar residents is one of the driving factors in our success story." After the success of the International Biobanking Conference 2019, the future will build further successful collaborations with our partners, Prof. Afifi affirmed.

\section{Author Disclosure Statement}

No conflicting financial interests exist.

Address correspondence to: Nahla Afifi, $M D, P h D$ Qatar Biobank Qatar Foundation Hamad Medical City, Building 317, P.O. Box 5825 Doha 9744 Qatar

E-mail: nafifi@qf.org.qa

\footnotetext{
${ }^{38}$ Director of Data-Intensive Computing at CRS4, Italy.

${ }^{39} \mathrm{Head}$ of Informatics Infrastructure at Genomics England, United Kingdom.

${ }^{40}$ Scientist with Qatar Genome Project, Qatar.

${ }^{41}$ Principal Investigator in Bioinformatics at Sidra Medicine and Adjunct Associate Professor at the Hamad Bin Khalifa University, Qatar.
} 